\title{
THE Q0957+561 TIME DELAY
}

\author{
RUDOLPH E. SCHILD \\ Harvard-Smithsonian Center for Astrophysics \\ 60 Garden Street, Cambridge MA 02138
}

\author{
AND \\ DAVID J. THOMSON \\ ATET Bell Labs \\ Murray Hill, NJ 07974-2079
}

\begin{abstract}
The Q0957+561 time delay remains controversial, and we present four new time delay calculations based upon the intensively sampled data of the last three years.
\end{abstract}

To avoid problems due to sampling in time delay estimation, the TwQSO was scheduled for observations each night of the 9-month observing window that begins on Oct. 1, and on average 130 nights of data have been obtained each season for the past three years.

In Figure 1 we show the data plotted for a 404-day time delay, but with no other alterations. The crosses are for image $A$, and the open squares are for image B. Note that the B brightness record extends to JD 249500 only, because of the time delay correction. The $\mathrm{B}$ magnitudes have been shifted with a small magnitude offset to show the agreement with the correctly plotted A data. A 0.1 magnitude brightness increase was seen near JD2948950, and a complex brightness change with decreasing, increasing, and then decreasing flux was seen near JD2949400. These features repeated 1.1 years later in the B image, and give a secure time delay signature.

In Figure 2 we show four cross-correlation calculations for just the 3year data subset shown in Figure 1. All four calculations peak near 404 days, and show no correlation amplitude for the alternative 540 day value. Of particular interest is the approximately 100 day widths of the symmetrical parts of the peaks, since one would have expected much sharper autocorrelation peaks with daily sampled data. 


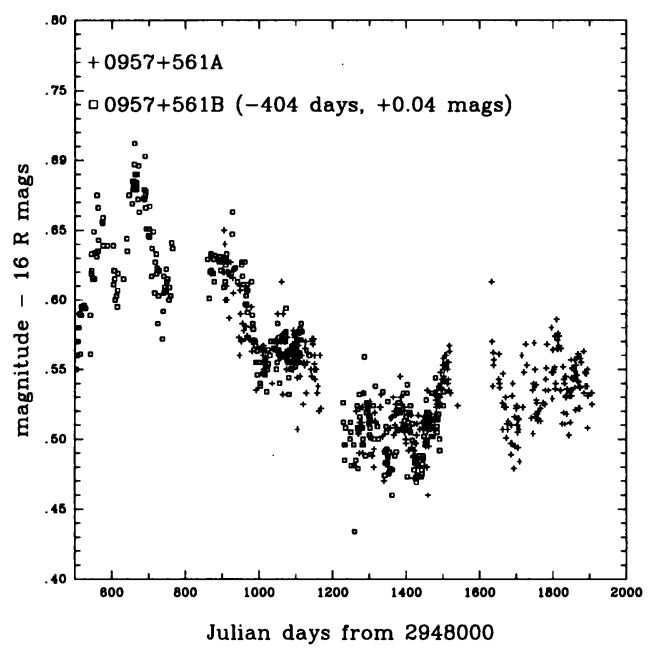

Figure 1. The Q0957+561 data plotted for a 405-day time delay.

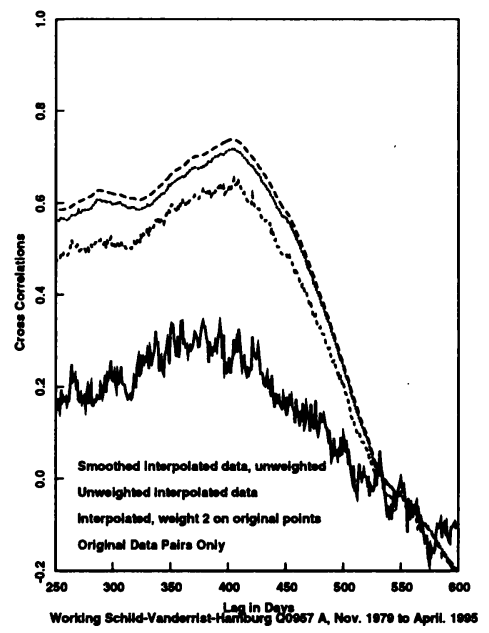

Figure 2. Four autocorrelation calculations for the 3-year data subset

The upper curve in Figure 2 is a autocorrelation for the 3-year subsample with 7-day median smoothing filter first applied to the data, giving a cross-correlation peak of 0.73 for a 404-day lag. If the data are not smoothed, the curve of connected dots shows a slightly lower level of cross-correlation and a peak for 405 days. Attaching double weight to the real data points in the interpolated data set produces a 405-day peak lag and lower level of correlation (third curve from top). The Edelson-Krolick method produces a shorter, 360-day peak and a lower amplitude (solid curve). 